\title{
The measurement of absolute reactor neutrino flux and spectrum, and their evolution at Daya Bay
}

\author{
Bei-Zhen Hu* on behalf of Daya Bay Collaboration \\ National Taiwan University, Taipei \\ E-mail: beizhenhu@hep1.phys.ntu.edu.tw
}

\begin{abstract}
The Daya Bay Reactor Neutrino Experiment consists of eight functionally identical detectors placed underground at different baselines from six $2.9 \mathrm{GW}_{t h}$ reactor cores. Since Dec. 2011, the experiment has collected more than 2.2 million inverse beta decay (IBD) candidates, enabling a precision measurement of the absolute reactor antineutrino flux and spectrum, and their fueldependent evolution. The comparison between measured spectrum and predictions from HuberMueller model revealed a $2.9 \sigma$ deviation for the whole energy region and mostly pronounced in the region around 4-6 MeV. The measurement of the evolution of the reactor antineutrino flux and spectrum showed a $2.8 \sigma$ discrepancy in the antineutrino flux variation with respect to the reactor fuel composition. The discrepancy suggests an overestimation of the predicted contribution from the ${ }^{235} \mathrm{U}$ fission isotope and indicates that this isotope could be primary contributor to the reactor antineutrino anomaly.
\end{abstract}

XXXIX International Conference on High Energy Physics (ICHEP2018)

4-11 July 2018

Seoul, Korea

${ }^{*}$ Speaker. 


\section{Introduction}

Electron antineutrinos, $\bar{v}_{e}$, are from the beta decay chains following fission processes. On average, $\sim 6 \bar{v}_{e}$ are released per fission with $\sim 2 \times 10^{20} \bar{v}_{e}$ per second for each GW of thermal power. The Daya Bay experiment has a powerful electron antineutrino source, the six reactor cores providing $17.4 \mathrm{GW}$ of thermal power. With optimized baselines, high statistics and a suitable measurement strategy (relative measurement), the experiment measured the most precise mixing angle $\theta_{13}$ [1] [2] [3]. The high statistics also give a door for the Daya Bay experiment to measure the flux and spectrum of antineutrinos and confirm the observed discrepancy of $\sim 6 \%$ between the prediction and past experimental results.

Before 2011, the antineutrino spectrum prediction was based on the beta decay spectra of the fission isotopes, ${ }^{235} \mathrm{U},{ }^{239} \mathrm{Pu}$ and ${ }^{241} \mathrm{Pu}$, measured by Institut Laue-Langevin (ILL) reactor experiment and the theoretical calculation of ${ }^{238} \mathrm{U}$ from P. Vogel (referred to as ILL+Vogel model). It was consistent with the experimental data. However, after 2011, the prediction was re-calculated by Huber and Mueller with improved theoretical methods (referred to as Huber+Mueller model). The new prediction is higher than the measured. This discrepancy is known as the "Reactor Antineutrino Anomaly".

\section{Improved detection efficiency estimation}

Recently, an extensive calibration campaign was completed by the Daya Bay experiment. Two neutron source, ${ }^{241} \mathrm{Am}-{ }^{13} \mathrm{C}$ and ${ }^{241} \mathrm{Am}-{ }^{9} \mathrm{Be}$, were deployed at different positions into a nearsite antineutrino detector. In total, the calibration data were collected from 59 differenct source and location points. A benchmark quantity was defined by $F=N([6,12] \mathrm{MeV}) / N([1.5,12] \mathrm{MeV})$, where $N([6,12] \mathrm{MeV})$ and $N([1.5,12] \mathrm{MeV})$ is the number of events with reconstructed delayed energy in the range of $N([6,12] \mathrm{MeV})$ and $N([1.5,12] \mathrm{MeV})$, respectively. A regression function for neutron detection efficiency is defined as $\varepsilon_{n}=c_{i}\left(F_{\text {data }, i}-F_{M C, b e s t, i}\right)+\varepsilon_{M C, b e s t} . \varepsilon_{M C, b e s t}$ is the neutron detection efficiency given by the best Daya Bay Monte Carlo (MC) model. $c_{i}$ describes the relation between $\varepsilon_{n}$ and $F_{i}$, and can be estimated through a linear regression from $20 \mathrm{MC}$ models. The results are in good agreement between calibration data and simulation on $F$ as shown in Fig. 7 of Ref. [6]. In addition, a variety of simulation models were studied at Daya Bay with a good agreement on the energy spectrum. The determination of the neutron detection efficiency has improved with the extensive calibration studies.

\section{Antineutrino measurements: Flux, Spectrum and Fuel Evolution}

There are two methods for the absolute reactor antineutrino flux measurement. In the first method, we used the data from all antineutrino detectors and determined the $\sin ^{2} \theta_{13}$ and the flux normalization $\mathrm{R}$ simultaneously with a $\chi^{2}$ function in Ref. [5]. In the second method, we only used two near site detectors and the measured value of $\sin ^{2} \theta_{13}$ to measure the IBD yield. The result of absolute antineutrino flux $\sigma_{f}$ is $(5.91 \pm 0.09) \times 10^{-43} \mathrm{~cm}^{2} /$ fission [6]. The ratio of the data and Huber+Muller's prediction is $R=0.952 \pm 0.014$ (exp.) \pm 0.023 (model) [6]. The Daya Bay result is consistent with others, as shown in Figure 1. Comparing with the prediction, the theoretical model is $5 \%$ higher. 


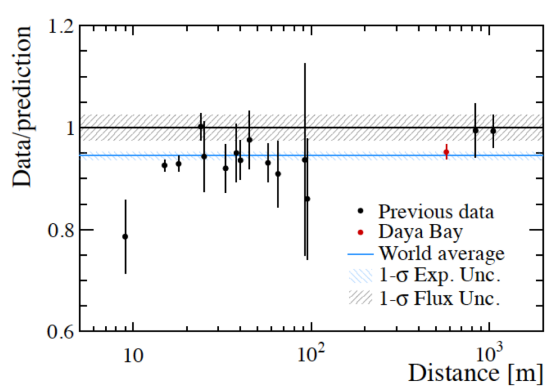

Figure 1: The measured reactor $\bar{v}_{e}$ rate as a function of the distance from the reactor, normalized to the theoretical prediction of Huber+Mueller model. [5]

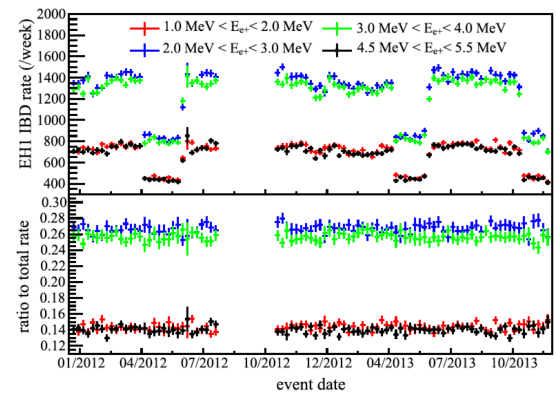

Figure 2: Top: The IBD event rates in the region of the excess (4.5 MeV $<\mathrm{E}_{e^{+}}<5.5 \mathrm{MeV}$, black) and outside this region. Bottom: The fraction ratio to total rate. [5]

The predicted and measured prompt energy spectra were shown in Fig. 23 of Ref. [5]. A clear bump from 4-6 MeV is shown when compared to Huber+Mueller model, local significance $4.4 \sigma$. Comparison to ILL+Vogel model gives similar bump. To verify the relation between the bump from 4-6 MeV and the antineutrino flux, the histories of IBD rate and the ratio to total rate were selected with different energy ranges, shown in Figure 2. The results point out that there is a clear correlation with reactor thermal power.

The Daya Bay experiment also measured the generic reactor antineutrino spectrum, shown in Fig. 23 of Ref. [5]. It was extracted by the singular value decomposition (SVD) regularization method and Bayesian iterative method. The spectrum is independent of the Daya Bay detector response and can be used for other reactor experiments, with small correction to different fission fractions.

There are some correlations between reactor fuel evolution and the reactor antineutrino flux and spectrum. To examine these, the effective fission fractions $F_{i}(t)$ can be defined as

$$
F_{i}(t)=\sum_{r=1}^{6} \frac{W_{t h, r}(t) \bar{p}_{r} f_{i, r}(t)}{L_{r}^{2} \bar{E}_{r}(t)} / \sum_{r=1}^{6} \frac{W_{t h, r}(t) \bar{p}_{r}}{L_{r}^{2} \bar{E}_{r}(t)}
$$

where $W$ is the thermal power, $p$ is the survival probability and $L$ is the baseline over 6 reactor cores. The effective fission fractions $F_{239}$ were divided into eight bins of differing fuel composition, shown in the Fig. 1 of Ref. [6].

Figure 3 shows the IBD yield per fission, $\sigma_{f}$, versus the effective ${ }^{239} \mathrm{Pu}$ fission fractions, $F_{239}$. The data points were fit with

$$
\sigma_{f}\left(F_{239}\right)=\bar{\sigma}_{f}+\frac{d \sigma_{f}}{d F_{239}}\left(F_{239}-\bar{F}_{239}\right)
$$

where $\overline{\sigma_{f}}$ is the total $F_{239}$-average IBD yield and $\bar{F}_{239}$ is the average ${ }^{239} \mathrm{Pu}$ fission fraction. The total IBD yield $\overline{\sigma_{f}}$ is determined to be $(5.91 \pm 0.09) \times 10^{-43} \mathrm{~cm}^{2} /$ fission, where the predicted $\overline{\sigma_{f}}$ is $(6.22 \pm 0.14) \times 10^{-43} \mathrm{~cm}^{2} /$ fission.

Assuming the IBD yields of the two minor isotope ${ }^{238} \mathrm{U}$ and ${ }^{241} \mathrm{Pu}$ from model prediction with an enlarged $10 \%$ error, the combined fit of the two major fission isotope ${ }^{235} \mathrm{U}$ and ${ }^{239} \mathrm{Pu}$, shown 
in Figure 4, were found to be $(6.17 \pm 0.17)$ and $(4.27 \pm 0.26) \times 10^{-43} \mathrm{~cm}^{2} /$ fission, respectively. These results also point out the $2.8 \sigma$ and $3.2 \sigma$ discrepancy in the antineutrino flux variation with respect to the reactor fuel composition. [4]

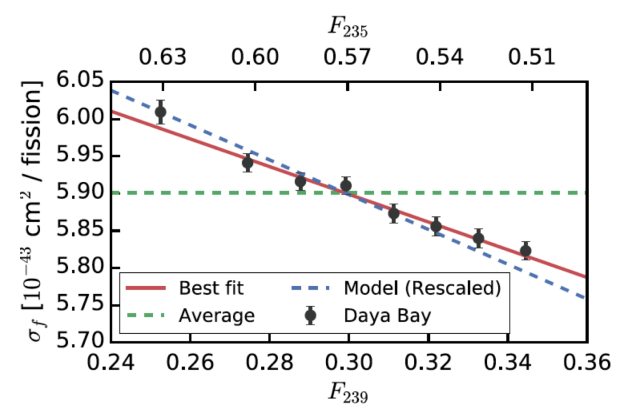

Figure 3: The IBD yield per fission, $\sigma_{f}$, versus the effective ${ }^{239} \mathrm{Pu}$ fission fractions, $F_{239}$. Red line is the best fit and blue one is the predicted yields from the Huber-Mueller model. [4]

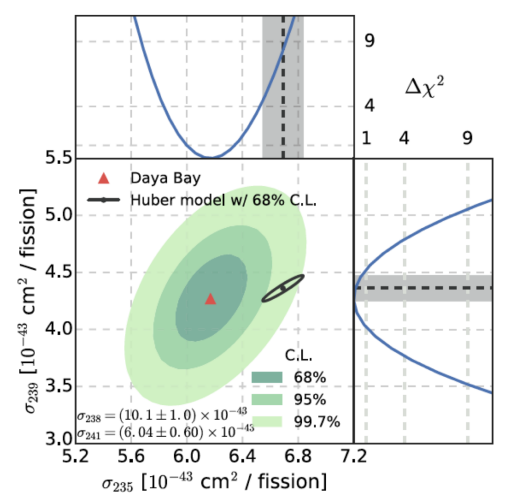

Figure 4: Combined measurement of IBD yields per fission for ${ }^{235} \mathrm{U}$ and ${ }^{239} \mathrm{Pu}, \sigma_{235}$ and $\sigma_{239}$. [4]

\section{Summary}

The Daya Bay experiment improved the neutron detection efficiency from $2.1 \%$ to $1.5 \%$. The absolute antineutrino flux $\sigma_{f}$ is $(5.91 \pm 0.09) \times 10^{-43} \mathrm{~cm}^{2} /$ fission. The ratio of the data and Huber+Muller's prediction is $R=0.952 \pm 0.014$ (exp.) \pm 0.023 (model). The absolute positron spectral shape is NOT consistent with the prediction. A bump is observed between 4-6 MeV with a $4.4 \sigma$ discrepancy. Our reactor fuel evolution study suggests that ${ }^{235} U$ is the main contributor to the reactor anomaly.

\section{References}

[1] F. P. An et al. [Daya Bay Collaboration], Phys. Rev. Lett. 108, 171803 (2012)

[2] F. P. An et al. [Daya Bay Collaboration], Phys. Rev. D 95, no. 7, 072006 (2017)

[3] D. Adey et al. [Daya Bay Collaboration], arXiv:1809.02261 [hep-ex].

[4] F. P. An et al. [Daya Bay Collaboration], Phys. Rev. Lett. 118, no. 25, 251801 (2017)

[5] F. P. An et al. [Daya Bay Collaboration], Chin. Phys. C 41, no. 1, 013002 (2017)

[6] D. Adey et al. [Daya Bay Collaboration], arXiv:1808.10836 [hep-ex]. 